\title{
Endothelial nitric oxide synthase in hypoxic newborn porcine pulmonary vessels
}

\author{
A A Hislop, D R Springall ${ }^{\dagger}$, H Oliveira, J S Pollock, J M Polak, S G Haworth
}

\begin{abstract}
Aims-To determine if the failure of neonatal pulmonary arteries to dilate is due to a lack of nitric oxide synthase (NOS).

Methods-A monoclonal antibody to endothelial NOS was used to demonstrate the distribution and density of NOS in the developing porcine lung after a period in hypobaric hypoxia. Newborn piglets were made hypertensive by exposure to hypobaric hypoxia $(50.8 \mathrm{kPa})$ from $<5$ minutes of age to 2.5 days of age, 3-6 days of age or 14-17 days of age. A semiquantitative scoring system was used to assess the distribution of endothelial NOS by light microscopy.
\end{abstract}

Results-NOS was present in the arteries in all hypoxic animals. However, hypoxia from birth caused a reduction in NOS compared with those lungs normal at birth and those normal at 3 days. Hypoxia from 3-6 days led to a high density of NOS compared with normal lungs at 6 days. Hypoxia from 14-17 days had little effect on the amount of NOS. On recovery in room air after exposure to hypoxia from birth there was a transient increase in endothelial NOS after three days of recovery, mirroring that seen at three days in normal animals.

Conclusions-Suppression of NOS production in the first few days of life may contribute to pulmonary hypertension in neonates.

(Arch Dis Child 1997;77:F16-F22)

Keywords: pulmonary circulation; nitric oxide synthase; hypoxia; endothelium; piglets

Pulmonary hypertension is a major cause of mortality and morbidity in the newborn period and is characterised by failure of the pulmonary arteries to dilate. This is normally accompanied by changes in endothelial and smooth muscle cell shape and cytoskeletal composition. ${ }^{1}$ The mechanisms causing these changes are not completely understood, but endogenous nitric oxide produced from L-arginine by nitric oxide synthase (NOS) probably has an important role. The type III (endothelial) form of NOS is present in normal porcine pulmonary vessels at birth and increases to a maximum at 2-3 days of age before reducing to the lower adult level. ${ }^{2}$ A postnatal decrease has also been described in sheep and rats. $^{34}$
Hypoxia is a common cause of failure of the pulmonary arteries to dilate. The pulmonary arteries of newborn piglets made hypertensive by exposure to hypobaric hypoxia for three days showed a loss of endothelium dependent, acetylcholine induced vasodilatation, but continued to dilate in response to nitric oxide, although this response was attenuated. ${ }^{5}$ Inhaled NO reduces pulmonary artery pressure in hypertensive 1 to 2 day old piglets, ${ }^{6}$ and is effective in the treatment of hypoxic newborn babies. ${ }^{78}$ Failure to dilate in hypoxic newborn humans and animals might be attributable to a reduction in the amount of endothelial NOS.

This study aimed to determine whether there were changes in the amount of endothelial NOS in the presence of pulmonary hypertension after hypobaric hypoxic exposure in the newborn period, and also to determine whether any changes were reversible on return to normoxia.

\section{Methods}

Large White piglets delivered naturally at term were used. Animals were randomly allocated to experimental groups as they were delivered. Fifty five animals were exposed to hypobaric hypoxia $(50.8 \mathrm{kPa})$ which gives an $\mathrm{FIO}_{2}$ of 0.096 either from birth ( $<5$ minutes old) for 2.5 days, or kept with their mother for three or 14 days and then exposed to hypoxia for three days. The newborn babies were warm and pink as they were put in the chamber. During exposure to hypobaric hypoxia, measurement with a pulse oximeter on the foreleg showed that the animals had a systemic arterial oxygen saturation of $71 \%$ with a standard deviation of $5 \%$. Eighteen animals were killed at the end of hypoxic exposure ( $n=6$ for each group). These animals had evidence of pulmonary hypertension with a decreased heart:weight ratio and increased arterial wall thickness, which has been reported before. ${ }^{9}$ The remaining 27 hypoxic animals were allowed to recover in room air with the sow and littermates for one, three, or six days before sacrifice. The animals were kept in the hypobaric chamber on straw, at a temperature of $25^{\circ} \mathrm{C}$ with a continuous supply of milk and mashed feed. The chamber is purpose built of steel, one metre in diameter and 1.5 metres long. It has a perspex viewing window which also allows entry for a temperature probe and a light source. The pressure is reduced by a vacuum pump with a flow of air through the chamber due to a controlled leak. This gives a flow of at least 30 litres per minute 
Table 1 Mean (SD) percentage of arteries containing different amounts of eNOS within endothelial cells during hypoxia

\begin{tabular}{|c|c|c|c|c|c|c|}
\hline Artery level & 3 day $C$ & $0-2.5 d H$ & 6 day $C$ & 3-6 day $H$ & 17 day $C$ & 14-17 day $H$ \\
\hline \multicolumn{7}{|l|}{ Bronchiolus } \\
\hline 0 & $0.6(1.3)$ & $5.0(8.1)$ & $15 \quad(8.7)$ & $3.3(4.5)$ & $15.6(12.3)$ & $5.7(4.4)$ \\
\hline $1-50$ & $18.6(17.4)$ & $38.5(11.9)$ & $43.1(12.5)$ & $23.7(15)$ & $40.5(19.4)$ & 30.5 (13.7) \\
\hline $51-100$ & $80.8(16.1)$ & $53.1(11.9)^{\star}$ & $40.3(15.3)$ & $72.9(18.6)^{\star}$ & $44 \quad(26.2)$ & $63.8(15.6)$ \\
\hline \multicolumn{7}{|c|}{ Terminal bronchiolus } \\
\hline 0 & $0.8(1.8)$ & $26.6(16.6)$ & $13.2(9.1)$ & $4.7(7.2)$ & $30.5(28.9)$ & $10.5(11.6)$ \\
\hline $1-50$ & $36.8(13.4)$ & $52.3(7.9)$ & $63.7(12)$ & $31.8(19.9)$ & $46 \quad(23.4)$ & $51.1(11.7)$ \\
\hline $51-100$ & $62.4(14)$ & $21(14.2)^{\star}$ & $23.1(16.1)$ & $63.6(21.6)^{\star}$ & $23.5(28.3)$ & $38.3(19.9)$ \\
\hline \multicolumn{7}{|c|}{ Respiratory bronchiolus } \\
\hline 0 & $1.2(2.7)$ & $35.1(26.7)$ & $16.8(9.4)$ & $4.8(7.6)$ & $31.3(20.1)$ & $27.8(11.9)$ \\
\hline $1-50$ & $41.4(4.3)$ & $51.3(17.7)$ & $56.7(13.7)$ & $38.8(18.3)$ & $44.3(10.2)$ & $52.1(8.9)$ \\
\hline $51-100$ & $57.4(4.3$ & $13.1(16.6)^{\star}$ & $26.5(17.6)$ & $56.3(23.2)$ & $24.3(16.9$ & $20 \quad(16.3)$ \\
\hline \multicolumn{7}{|c|}{ Alveolar duct } \\
\hline 0 & $4.8(5.9)$ & $50.1(13.3)$ & $34.3(20.8)$ & $10.7(11.1)$ & $51.7(16.4)$ & $55 \quad(16.4)$ \\
\hline $1-50$ & $37.8(11.4)$ & $48.1(13.1)$ & $46 \quad(16.4)$ & $34.3(9.9)$ & $34.8(14.6)$ & $36.5(12.5)$ \\
\hline $51-100$ & $57.4(14.5)$ & $1.7(2.9)^{\star}$ & $19.7(12.2)$ & $55(17.04)^{\star}$ & $13.5(11.1)$ & $8.5(4.9)$ \\
\hline \multicolumn{7}{|l|}{ Alveoli } \\
\hline 0 & $9.6(17.3)$ & $98.1(2.9)$ & $3.7(16.2)$ & $30 \quad(32.7)$ & 100 & 100 \\
\hline $1-50$ & $33.8(19.7)$ & $1.8(2.9)^{\star}$ & $9.5(12.6)$ & $30.5(24.01)$ & 0 & 0 \\
\hline $51-100$ & $56.6(23.8)$ & $0^{\star}$ & $3.5(8.6)$ & $39.5(25.5)^{\star}$ & 0 & 0 \\
\hline
\end{tabular}

$\star \mathrm{P}<0.05$ in comparison with age-matched controls.

and maintains the carbon dioxide at less than $0.3 \%$. The chamber was cleaned and the supplies replenished twice a day. The animals kept in the chamber from birth were tube fed at this time so that the pressure was normal for 30 minutes. Twenty four control animals aged newborn, 3 days, 6 days and 17 days were also studied, and these animals were maternally fed. For each group of control and experimental animals, piglets were taken from different litters so that animals from at least three different litters were used in each group. Littermates were used for some control and experimental groups, but not within the same group.

This investigation conforms with the Guide for the care and use of laboratory animals published by the US National Institutes of Health (NIH publication No 85-23, revised 1985). Animals were killed in accordance with British Home Office regulations and as approved by the Animals Ethics Committee of the Institute of Child Health.

Immediately after death transverse slices of tissue from the mid-lung region were immersed in freshly defrosted $1 \%$ paraformaldehyde solution in phosphate buffered saline (PBS: $0.01 \mathrm{M}$ phosphate buffer, $\mathrm{pH} 7.4$, with $0.15 \mathrm{M}$ $\mathrm{NaCl}$ ) for four hours and then washed in PBS containing $10 \%$ sucrose for at least 24 hours.

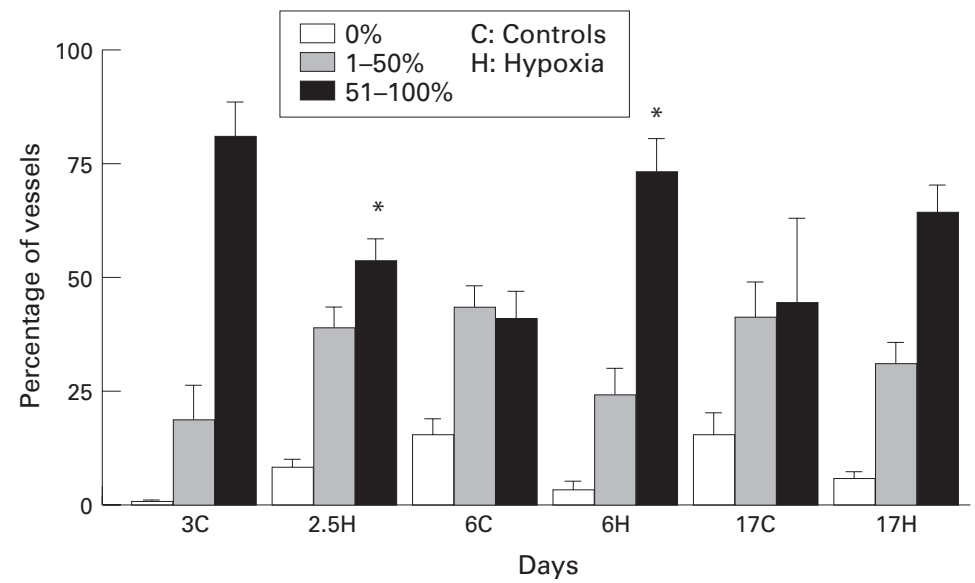

Figure 1 Mean (SD) percentage of arteries at bronchiolar level containing no endothelial NOS, containing up to $50 \%$, or $51-100 \%$ in hypoxic and control piglets. ${ }^{\star} P<0.05$ compared with controls of the same age.
After snap freezing, sections $7 \mu \mathrm{m}$ thick were cut at $21^{\circ} \mathrm{C}$ in a cryostat, and pairs of serial sections stained either with haematoxylin and eosin or for immunochemistry using a mouse monoclonal antibody to constitutive NOS, purified from cultured bovine aortic endothelial cells, known to show immunoreactivity to porcine tissue. ${ }^{10}$ Sections were immersed in $0.03 \%$ hydrogen peroxide in methanol for 30 minutes to block endogenous peroxidase, followed by three 10 minute washes in PBS. Non-specific binding was blocked by incubation in 3\% normal horse serum for 20 minutes. Sections were then blotted to remove excess serum and incubated overnight with the monoclonal antibody diluted 1 in 25000 in PBS containing $0.05 \%$ bovine serum albumin and $0.1 \%$ sodium azide. After washing in PBS sections were successively incubated with biotinylated horse antiserum to mouse IgG (Vector Laboratories, Burlingame, CA, USA) diluted 1 in 100 in PBS with bovine serum albumin and freshly prepared avidinbiotinylated-peroxidase complex (Vector Laboratories) for 30 and 60 minutes, respectively. Peroxidase activity was demonstrated using the nickel enhanced diaminobenzidine method. ${ }^{11}$ Control sections were treated in the same way except that the primary antiserum was omitted.

The distribution of endothelial NOS in the pulmonary arteries and veins was examined using light microscopy. A semiquantitative method of assessing the amount of endothelial NOS was used. Arteries were identified according to their position within the lung. Five types of artery were identified: arteries accompanying bronchioli; arteries accompanying terminal bronchioli; arteries accompanying respiratory bronchioli; arteries accompanying alveolar ducts; and arteries accompanying alveoli. For each artery, the amount of endothelial NOS was classified as: none; up to $50 \%$ of the endothelial cells containing endothelial NOS; or $51-100 \%$ of the endothelial cells containing NOS. For each of the five types of artery the percentage falling into the three groups was determined. At least 20 arteries of each type were assessed in each animal and at least 50 in the alveolar duct and alveolar 

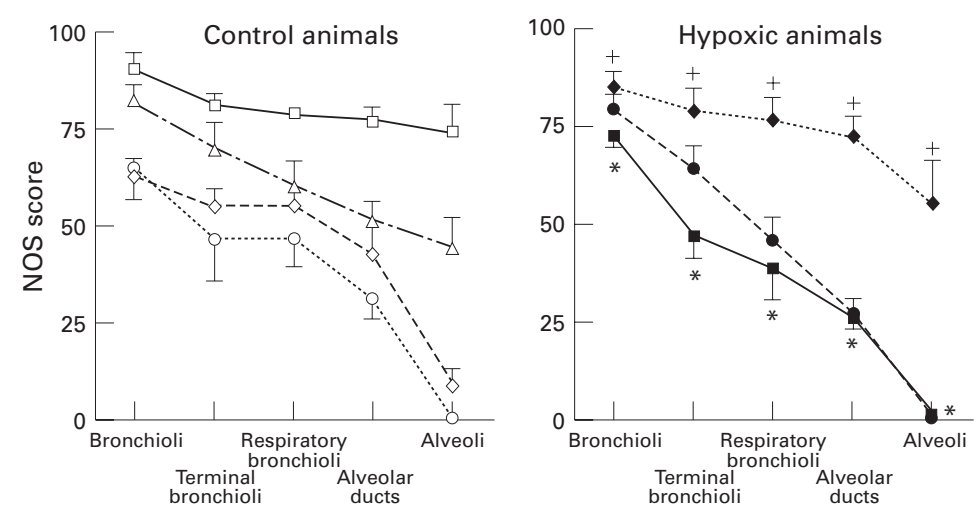

$$
\begin{aligned}
& --\triangle-\text { Newborn } \\
& \neg-3 \text { day control } \rightarrow 0-2.5 \text { day hypoxia } \\
& -\diamond-6 \text { day control }-\prec-\text { 3-6 day hypoxia } \\
& \text {...... } 17 \text { day control ...... 14-17 day hypoxia }
\end{aligned}
$$

Figure 2 NOS score (SD) for control and hypoxic animals in arteries at the level of the bronchioli, terminal bronchi, respiratory bronchioli, alveolar ducts, and alveoli. ${ }^{\star} P<0.05$ compared with control 3 day olds; $+P<0.05$ compared with control 6 day olds.

region. For each control and hypoxic group, and for each type of artery, the mean value of each of the three percentage groups was determined and the results compared using ANOVA and where appropriate Student's $t$ test with Bonferroni's correction for multiple comparisons. A P value of $<0.05$ was taken as showing a significant difference.

An average score for the amount of endothelial NOS in the arteries at each level was also derived from these data in order to help understanding. By multiplying the percentage of vessels with no endothelial NOS by 0 , vessels with $1-50 \%$ by 0.5 , and vessels with $51-100 \%$ by 1 and adding them together, a score out of 100 was produced for each type of artery. Thus if all arteries studied had $51-100 \%$ of their endothelial cells containing NOS, the score would be 100. A mean score for each type of artery in each age group was calculated. From these scores a mean score for the whole lung was also calculated. Results were compared using ANOVA and Student's $t$ test as above.

\section{Results}

Immunoreactivity for endothelial NOS was seen on the endothelial cells of pulmonary arteries and veins in all cases, as reported before. ${ }^{2}$ The amount of endothelial NOS staining was greater in larger and more proximal arteries than in the peripheral arteries in control, hypoxic, and recovery animals. The endothelial NOS immunoreactivity was similar in veins of all sizes and was not altered by hypoxia. In arteries changes were seen with hypoxia and during recovery.

In the arteries at bronchiolar level, in all normoxic piglets, some of the endothelial cells in most of the arteries contained endothelial NOS (fig 1) (table 1). The highest value of endothelial NOS was present at 3 days of age, when over half of the endothelial cells stained positively in $80 \%$ of bronchiolar arteries. The number of arteries with this large amount of NOS decreased in the older control animals (6 and 17 days).

In animals made hypoxic from birth the endothelial NOS immunoreactivity was reduced at bronchiolar level. Fewer arteries showed maximum staining $(\mathrm{P}<0.05)$ compared with normal lungs at 3 days (fig 1). By contrast, in those animals made hypoxic from 3 to 6 days, endothelial NOS immunoreactivity was increased. More arteries had endothelial NOS in $51-100 \%$ of their endothelial cells at 6 days $(\mathrm{P}<0.05)$ than did the normal lung at the same age. In those made hypoxic from 14-17 days, the amount of endothelial NOS was similar to that in normal animals of the same age. Thus in the arteries accompanying bronchioli the response to chronic hypobaric hypoxia varied according to the age at the onset of exposure. The same pattern of change was seen in arteries accompanying terminal bronchioli, respiratory bronchioli, alveolar ducts and alveoli (table 1). In the smallest arteries, those accompanying the alveoli, there was almost no staining in animals exposed to hypoxia from birth, while in the normal lungs at 3 days over half of the arteries had $51-100 \%$

\begin{tabular}{|c|c|c|c|c|c|c|c|c|c|}
\hline Artery level & $0-2.5 H+1 R$ & $0-2.5 H+3 R$ & $0-2.5 H+6 R$ & $3-6 H+1 R$ & $3-6 H+3 R$ & $3-6 H+6 R$ & $14-17 H+1 R$ & $14-17 H+3 R$ & $14-17 H+6 R$ \\
\hline \multicolumn{10}{|c|}{ Bronchiolus } \\
\hline 0 & $15 \quad(1.4)$ & $7 \quad(10.4)$ & $15.5(6.4)$ & $14 \quad(15.6)$ & $16 \quad(7.2)$ & $19.8(14.9)$ & $8.5(2.1)$ & $15.7(5.1)$ & $(7.8)$ \\
\hline $1-50$ & $51 \quad(9.9)$ & $24.3(21.8)$ & $52 \quad(16.04)$ & $42 \quad(21.2)$ & $42 \quad(4.4)$ & $44.2(21.5)$ & $37 \quad(5.6)$ & $43.3(9.9)$ & $45.5 \quad(5.1)$ \\
\hline $51-100$ & $34 \quad(8.4)$ & $68.7(30.5)$ & $43.5(36.1)$ & $43.5(36.1)$ & $42 \quad(10.5)$ & $36 \quad(35.2)$ & $54.5(7.8)$ & $41 \quad(7.5)$ & $41.5 \quad(5)$ \\
\hline \multicolumn{10}{|c|}{$\begin{array}{l}\text { Terminal } \\
\text { bronchiolus }\end{array}$} \\
\hline 0 & $22 \quad(12.7)$ & $8.3(9.6)$ & $22.5(8.3)$ & $16 \quad(15.6)$ & $28 \quad(30.3)$ & $23.7(20.4)$ & $14.5(7.8)$ & $29.7(19.1)$ & $(13.1)$ \\
\hline $1-50$ & $60 \quad(14.1)$ & $45.3(23.0)$ & $60.2(11.6)$ & $44 \quad(12.7)$ & $45.7(8)$ & $47.8(11.6)$ & $29 \quad(15.6)$ & $54.3(20.8)$ & $60.25(14.5)$ \\
\hline $51-100$ & $18 \quad(1.4)$ & $45.7(28.0)$ & $17.2(13.6)$ & $40 \quad(28.2)$ & $26 \quad(22.7)$ & $24.5(29.4)$ & $56.5(27.3)$ & $17.7(18.1)$ & $24.75(22.4)$ \\
\hline \multicolumn{10}{|c|}{$\begin{array}{l}\text { Respiratory } \\
\text { bronchiolus }\end{array}$} \\
\hline 0 & $28.5(10.6)$ & $6.7(5.9)$ & $39.7(10.3)$ & $22 \quad(19.8)$ & $27.7(18.6)$ & $27 \quad(18.2)$ & $14.5(7.8)$ & $21.3(7.2)$ & $24.25(12.6)$ \\
\hline $1-50$ & $70 \quad(8.4)$ & $54.3(21.8)$ & $47.2(11.4)$ & $53 \quad(15.5)$ & $51.3(14.8)$ & $47.5(14.4)$ & $58.5(7.8)$ & $70.7(6.7)$ & $50.75(11.5)$ \\
\hline $51-100$ & $1.5(2.1)$ & $39 \quad(2.7)$ & $13 \quad(9.3$ & $25 \quad(35.3)$ & $25 \quad(35.3)$ & $24.7(32.9)$ & $27 \quad(0)$ & $8 \quad(7)$ & $24.5 \quad(14.1)$ \\
\hline \multicolumn{10}{|c|}{ Alveolar duct } \\
\hline 0 & $49 \quad(1.4)$ & $9 \quad(5.2)$ & $56.2(31.5)$ & $41.5(12.02)$ & $38.5(7.6)$ & $36.2(17.1)$ & $35 \quad(7.1)$ & $49.7(18.0)$ & $(26)$ \\
\hline $1-50$ & $41 \quad(5.3)$ & $46 \quad(14.4)$ & $37.2(27.9)$ & $31 \quad(19.8)$ & $50 \quad(3)$ & $47.8(15.1)$ & $44.5(10.6)$ & $43.7(10.1$ & (11.7) \\
\hline $51-100$ & $9.7(3.9)$ & $45 \quad(16.5)$ & $6.5(14.5)$ & $22.5(31.8)$ & $10.3(5.8)$ & $16 \quad(19.1)$ & $20.5(17.7)$ & $6.7(8.3)$ & $(16.3)$ \\
\hline \multicolumn{10}{|l|}{ Alveoli } \\
\hline 0 & 100 & $73.7(25.1)$ & & 100 & 100 & 100 & $77.5(31.8)$ & $90.3(16.7)$ & $92.5 \quad(5)$ \\
\hline $1-50$ & 0 & $21.3(25.7)$ & $2 \quad(4)$ & 0 & 0 & 0 & $22.5(31.8)$ & $9.7(16.7)$ & $7.5 \quad(5)$ \\
\hline $51-100$ & 0 & $4.7(8.1)$ & 0 & 0 & 0 & 0 & 0 & 0 & 0 \\
\hline
\end{tabular}
of the endothelial cells showing NOS immunoreactivity. For those animals made hypoxic

Table 2 Mean (SD) percentage of arteries containing different amounts of eNOS within endothelial cells

$\mathrm{H}=$ Days of hypoxia; $\mathrm{R}=$ days of recovery. 


\section{0-2.5 day hypoxia}
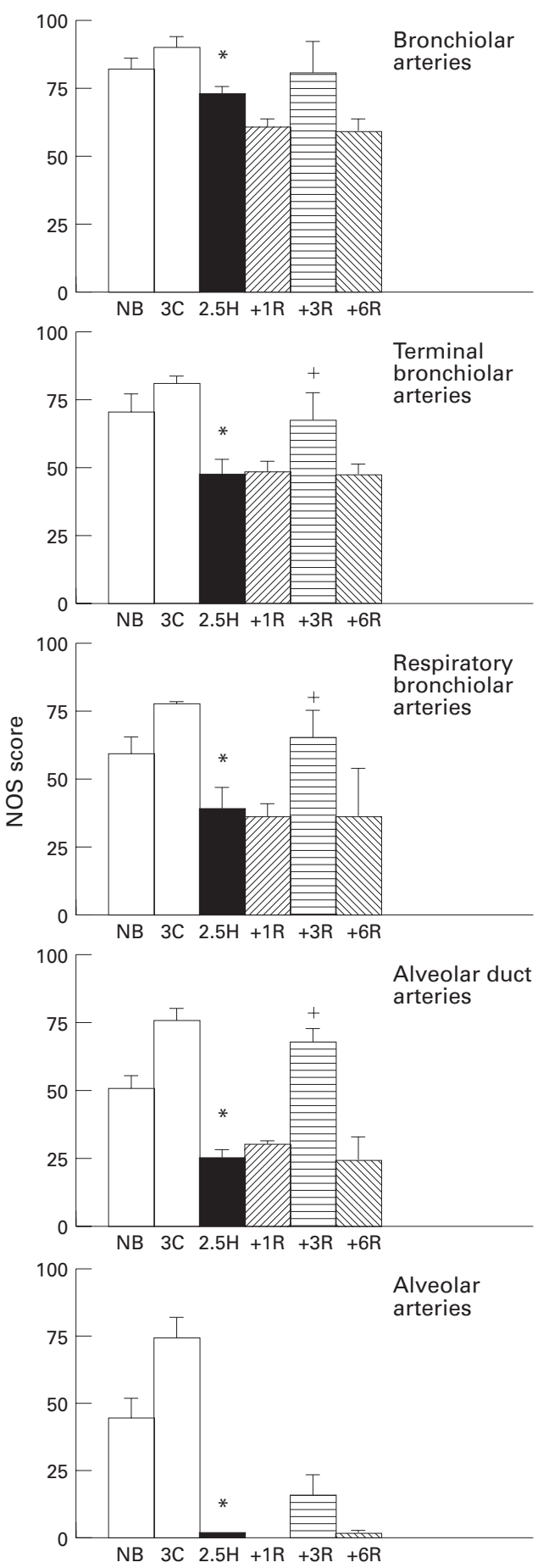

Figure 3 NOS score (SD) for control (C), hypoxic $(H)$, and recovery $(R)$ animals at each arterial level in $0-2.5$ day hypoxic group. ${ }^{\star} P<0.05$ compared with control 3 day olds; $+P<0.05$ compared with 2.5 day hypoxic animals.

from 3-6 days, at 6 days of age $40 \%$ of these arteries, rather than the normal $3.5 \%$, were immunoreactive for endothelial NOS in over half the endothelial cells.

The mean score for the amount of endothelial NOS in arteries at each level, in both the control and hypoxic animals, showed a decrease from proximal to distal arteries at all ages (fig 2). In the normal animals the score increased at 3 days $(\mathrm{P}<0.05)$, as reported
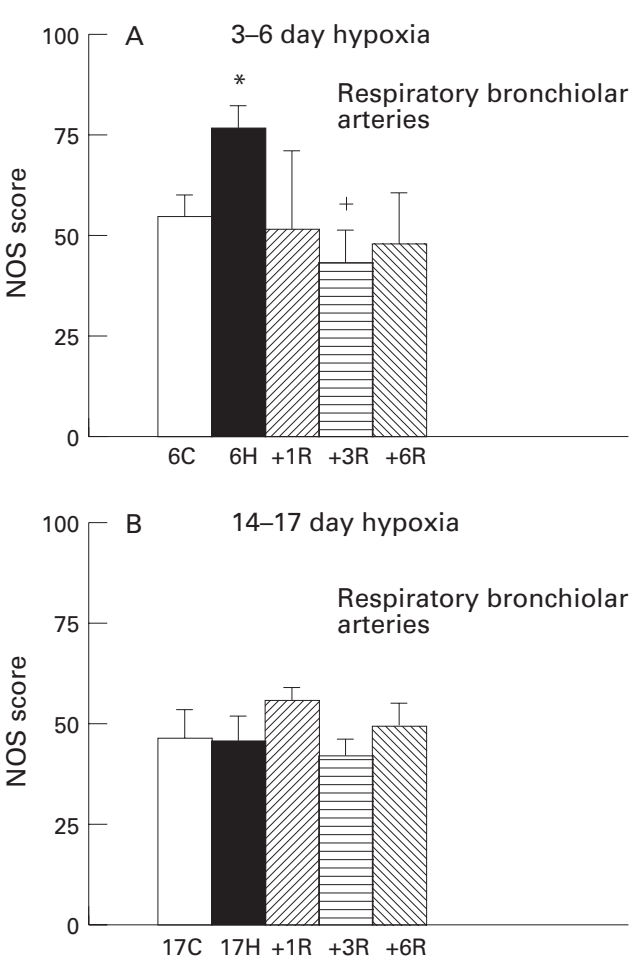

Figure 4 NOS score for control $(C)$, hypoxic $(H)$, and recovery $(R)$ animals at respiratory bronchiolar level in (A) 3-6 day hypoxic group and in (B) 14-17 day hypoxic group. ${ }^{\star} P<0.05$ compared with 6 day old controls; $+P<0.05$ compared with 6 day hypoxia.

before $^{2}$ (figs 2 and 3). After exposure to hypoxia from birth (fig 3 ) this increase was not seen and at the end of hypoxic exposure the NOS score was reduced compared with that in the control 3 day olds $(\mathrm{P}<0.05$ at all levels). The score was less than that seen at birth $(\mathrm{P}<0.05)$. After hypoxic exposure from 3-6 days, the score was higher than in the normal at 6 days of age ( $P<0.05$ at all levels) (figs 2 and 4A). After 14-17 days of hypoxia the score was not significantly different from control values at 17 days at any level (figs 2 and 4B).

After exposure to hypoxia from birth to 2.5 days there was no change in endothelial NOS staining after one day of recovery in room air, but after three days the staining was increased in vessels from bronchiolar to alveolar duct level (table 2 and fig 3). In these 6 day old animals the NOS score was significantly greater than immediately after hypoxia $(\mathrm{P}<0.05$ at terminal bronchiolar, respiratory bronchiolar, and alveolar duct levels) and only slightly less than the score in the normal at three days in arteries from bronchiolar to alveolar duct level. After 6 days of recovery, when the animals were 9 days old, the NOS score had decreased to to almost the level in the normal lung at 6-17 days of age.

On recovery from hypoxic exposure from 3-6 days the NOS score had decreased significantly by three days of recovery ( $\mathrm{P}<0.05$ for all levels), was close to the normal for age, and showed little further change (fig 4A). On recovery from 14-17 days of hypoxia (fig 4B), there were no significant changes in NOS score from normal, but there was some increase in endothelial NOS immunoreactivity after one day of recovery in that there were arteries at 


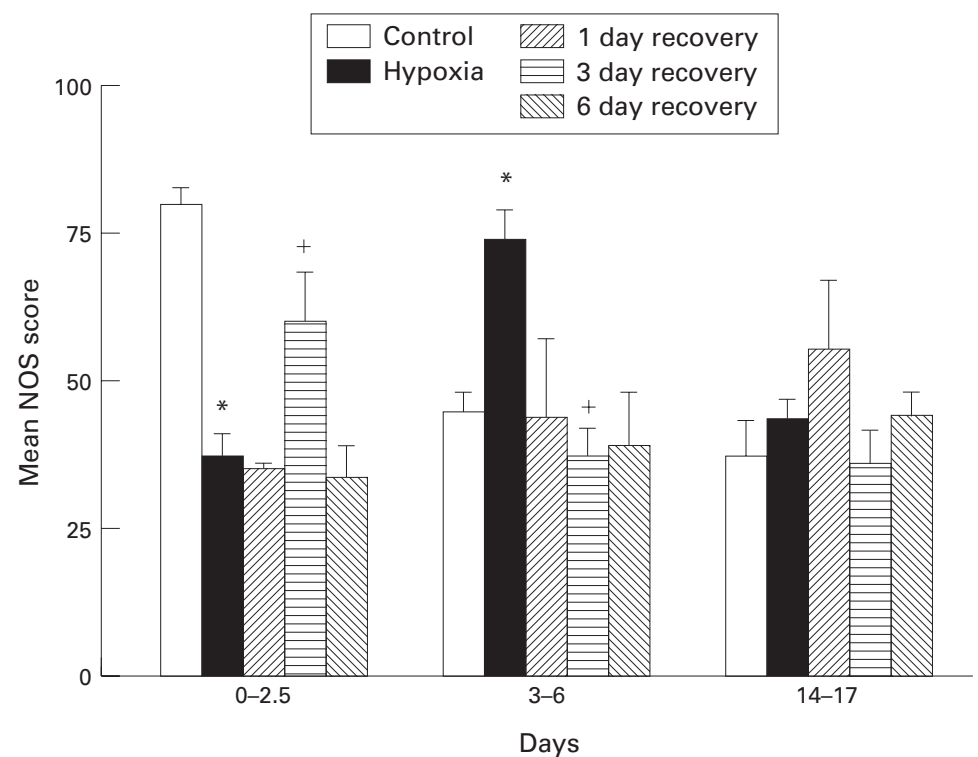

Figure 5 Mean (SD) NOS score for the three hypoxic groups. ${ }^{\star} P<0.05$ compared with controls of the same age; $+P<0.05$ compared with hypoxia.

alveolar level with endothelial NOS in their endothelial cells which is not a feature of the normal lung at this age (table 2).

Thus in animals exposed to hypobaric hypoxia from birth the overall amount of endothelial NOS, as shown by the mean NOS score for all arteries (fig 5), did not increase as it does in the normal lung immediately after birth $(\mathrm{P}<0.05$, three day control vs 2.5 days of hypoxia), but on recovery, a transient increase was seen after three days $(\mathrm{P}<0.05$, compared with hypoxia), mirroring that seen after three days in normal animals. The amount of endothelial NOS remained high in animals exposed to hypoxia from days 3 to 6 instead of decreasing as in normal animals, but after one day of recovery it had decreased to normal. Hypoxia had little effect on the amount of endothelial NOS in those animals exposed from 14-17 days when the normal mature low concentration of endothelial NOS had been reached before hypoxic exposure.

\section{Discussion}

In normal piglets more pulmonary arterial endothelial NOS is present at birth than is seen in adult pigs. The amount increases to reach a maximum at 2 to 3 days and then decreases during the next 10 days of life. ${ }^{2}$ The present study shows that exposure to chronic hypobaric hypoxia alters this pattern of change, the response varying according to the age at the onset of exposure to hypoxia. Pulmonary arterial endothelial NOS was always present, although it decreased in amount in animals exposed to hypoxia from birth. Thus the failure of endothelium dependent acetylcholine induced relaxation in the isolated pulmonary arteries of chronically hypoxic newborn piglets, studied using the same experimental model ${ }^{5}$ and in some instances the same lung as used in the present study, cannot be attributed to absence of endothelial NOS. In these physiological studies the endothelium independent relaxation to $\mathrm{NO}$ and Zaprinast was also impaired despite normal basal and stimulated generation of cGMP. Further studies are clearly indicated.

In the adult rat lung gene expression for endothelial NOS is upregulated in the alveolar arteries after three weeks of normobaric hypoxic exposure. ${ }^{12}$ Russ and Walker ${ }^{13}$ suggested that chronic hypoxia in adult rats enhanced the relaxant response to acetylcholine. Others have shown an increase in the vasoconstrictor response to the NOS inhibitor L-NAME, implying increased NO release, and an increase in NO products in the lung effluent after hypoxia. ${ }^{14}$ Other investigators report impaired acetylcholine induced relaxation following chronic hypoxic exposure in the adult lung, ${ }^{15-18}$ as did we in the newborn lung. ${ }^{5}$ This observation is not incompatible with the continued presence of endothelial NOS and even of its upregulation. The excessive contraction of pulmonary arterial smooth muscle cells in an hypoxic environment may stimulate excessive NO release. ${ }^{19}$ Shear stress will increase in contracted vessels and an increase in shear stress also increases NO production..$^{21}$ The balance of evidence suggests that NO production is at least normal during chronic hypoxia but that endothelium dependent relaxation is impaired. This suggests that other parts of the NO effector system are altered. Thus there may be a "threshold effect," the smooth muscle cells requiring a considerably greater amount of NO than the normal lung to effect relaxation, or there may be an increase in the breakdown of NO by oxygen free radicals. Inhalation of NO is effective in lowering the pulmonary arterial pressure in hypoxic newborn infants and in acutely hypoxic newborn piglets. ${ }^{68}$ A relative lack of the NO substrate L-arginine in animals not responding is suggested by studies which show a reduction in pulmonary arterial pressure in fetal sheep infused with L-arginine. ${ }^{22}$ The production of endothelial NOS and release of NO could be reduced if the endothelial cells were abnormal in structure after hypoxia. Ultrastructural studies have shown cytoskeletal abnormalities in enlarged endothelial cells in the newborn hypoxic piglet ${ }^{23}$ and the endothelial cells of hypoxic rats have an increased replication rate and an increase in cell size with changes in component parts. ${ }^{24}$ The endothelial cells of babies with chronic hypoxic lung disease $\mathrm{e}^{25}$ and those of adults living at high altitude also show morphological abnormalities. ${ }^{26}$

In the present study the amount of endothelial NOS staining in the pulmonary arteries at the end of hypoxic exposure depended on the age at onset of exposure. When exposed to hypoxia from birth, by 2.5 days there was less endothelial NOS than in normal newborn piglets. Fetal sheep also show a reduction in endothelial NOS when the animals were made pulmonary hypertensive in utero by ligation of the ductus arteriosus. ${ }^{27}{ }^{28}$ Thus unlike adult pulmonary arterial endothelium, fetal endothelium may be unable to maintain endothelial NOS synthesis in the presence of an exaggerated increase in pulmonary arterial pressure and the newborn piglet may have responded to 
chronic hypoxia as though it was still a fetus. Structurally, the porcine pulmonary arteries after 2.5 days of hypoxia resembled those of the fetus at term. ${ }^{923}$

In animals made hypoxic from days 3 to 6 , the endothelial NOS immunoreactivity was greater than in the normal animal at 6 days, and similar to that seen at the onset of exposure. Rather than there being an increase in endothelial NOS production, as in experimental adult animals, it seems more likely that exposure to chronic hypoxia prevented the reduction in endothelial NOS normally seen during this time interval. Exposure to hypoxia from days 14-17 had little effect in endothelial NOS immunostaining. The larger pulmonary arteries did show some increase in endothelial NOS immunostaining at 17 days, but the smaller arteries did not. By day 14, adaptation to extrauterine life is complete in the porcine lung and exposure for more than three days might have produced a response more similar to that seen in adult rats. ${ }^{12}$

The similarity of endothelial NOS immunostaining in the normal and hypoxic pulmonary veins suggest that venous endothelial NOS may be more stable than arterial endothelial NOS in the newborn porcine lung. Basal release of NO from the veins of newborn lambs is thought to make an important contribution to achieving and maintaining a low pulmonary vascular resistance after birth ${ }^{29}$ and the NOS inhibitor L-NAME has a greater effect on ovine pulmonary veins than on the pulmonary arteries $^{30}$ The production of endothelial NOS seems to be reduced in the pulmonary veins of the chronically hypoxic adult rat and ferret, ${ }^{31}{ }^{32}$ but the pharmacological response of the newborn porcine pulmonary veins to chronic hypoxia has not been studied.

Like the response to hypoxia, the response to recovery also varied according to the age of the animal at the onset of hypoxic exposure. In animals exposed to hypoxia from birth endothelial NOS immunostaining increased rapidly after three days of recovery in room air, the animals responding to room air as though they were newly born, because endothelial NOS immunostaining increases in normal piglets at 3 days of age. Endothelial NOS immunostaining then decreased to the lower level seen in older normal animals. Those exposed from 3-6 days of age showed a rapid reduction in endothelial NOS immunostaining to the normal lower level after one day of recovery and those exposed from 14-17 days showed little change in hypoxia or recovery.

The clinical implications of these findings are encouraging, suggesting as they do that babies who have sustained a perinatal hypoxic insult may retain endothelial NOS during the injury, although failure of endothelial NOS to rise normally in those exposed to hypoxia from birth may contribute to persistent pulmonary hypertension of the newborn. The surge in endothelial NOS production after oxygenation has been achieved is also encouraging. In animals first allowed to adapt to extrauterine life and then exposed to hypoxia at 3 days of age, endothelial NOS immunostaining re- mained as high during exposure to hypoxia as it was during the earlier period of postnatal adaptation. It is dangerous to extrapolate findings from an intrinsically healthy animal with normal lung parenchyma to sick babies with parenchymal lung disease, often born prematurely, but the findings support the present management policy of targeting the pulmonary arterial smooth muscle cells with nitric oxide until greater understanding of endothelial dysfunction offers additional, possibly alternative modes of therapy.

This work was supported by the British Heart Foundation.

1 Hall SM, Haworth SG. Normal adaptation of pulmonary arterial intima to extrauterine life in the pig: ultrastructural studies. F Pathol 1986;149:55-66.

2 Hislop AA, Springall DR, Buttery LDK, Pollock JS, Haworth SG. Abundance of endothelial nitric oxide synthase in newborn intrapulmonary arteries. Arch Dis Child 1995; 12:F17-21.

3 Halbower AC, Tuder RM, Franklin WA, Pollock JS, Forstermann U, Abman SH. Maturation-related changes in endothelial nitric oxide synthase immunolocalization in developing ovine lung. Am f Physiol 1994;11:L585-91.

4 North AJ, Star RA, Brannon TS, Ujiie K, Wells LB, Lowenstein CJ, et al. Nitric oxide synthase type I and type III gene expression are developmentally regulated in rat lung. Am 7 Physiol 1994;266:L635-41.

5 Tulloh RMR, Hislop AA, Boels PJ, Deutsch J, Haworth SG. Chronic hypoxia inhibits postnatal maturation of porcine intrapulmonary artery relaxation. $A m$ f Physiol 1997;272:H2430-5

6 Etches PC, Finer NN, Barrington KR, Graham AJ, Chan WKY. Nitric oxide reverses acute hypoxic pulmonary hypertension in the newborn piglet. Pediatr Res 1994;35:15-19.

7 Roberts JD, Polaner DM, Lang P, Zapol WM. Inhaled nitric oxide in persistent pulmonary hypertension of the oxide in persistent pulmonary

8 Kinsella JP, Neish SR, Shaffer E, Abman SH. Low-dose inhalational nitric oxide in persistent pulmonary hyperteninhalational nitric oxide in persistent pulmonary
sion of the newborn. Lancet 1992;340:819-20.

sion of the newborn. Lancet 1992;340:819-20.
9 Haworth SG, Hislop AA. Effect of hypoxia on adaptation of the pulmonary circulation to extra-uterine life in the pig. Cardiovasc Res 1982;16:293-303.

10 Pollock JS, Forstermann U, Mitchell JA, Warner TD, Schmidt HHHW, Nakane M, et al. Purification and characterisation of particulate endothelium-derived relaxing endothelial cells. Proc Natl Acad Sci USA 1991;88:104804.

11 Shu S, Ju G, Fan L. The glucose oxidase-DAB-nickel method in peroxidase histochemistry of the nervous system. Neurosci Lett 1988;85:169-71.

12 Le Cras TD, Xue C, Rengasamy A, Johns RA. Chronic hypoxia upregulates endothelial and inducible NO synthase gene and protein expression in rat lung. Am f Physiol 1996;270:L164-70

13 Russ R, Walker B. Maintained endothelium-dependent pulmonary vasodilatation following chronic hypoxia in the rat. $\mathcal{F}$ Appl Physiol 1993;74:339-44

14 Isaacson TC, Hampl V, Weir EK, Nelson DP, Archer SL. Increased endothelium-derived NO in hypertensive pulmonary circulation of chronically hypoxic rats. $\mathcal{f} A p p l$ Physiol 1994;76:933-40.

15 Adnot S, Raffestin B, Eddahibi S, Braquet P, Chabrier P-E. Loss of endothelium dependent relaxant activity in the pulmonary circulation of rats exposed to chronic hypoxia. f Clin Invest 1991;87:155-62.

16 Crawley DE, Zhao L, Giembycz MA, Liu S, Barnes P, Winter RJD, et al. Chronic hypoxia impairs soluble guanylyl cyclase-mediated pulmonary arterial relaxation in the rat. Am f Physiol 1992;263:L325-32.

17 Shaul PW, Wells LB, Horning KM. Acute and prolonged hypoxia attenuate endothelial nitric oxide production in hypoxia attenuate endothelial nitric oxide production in rat pulmonary arteries by differe
vasc Pharmacol $1993 ; 22: 819-27$.

18 Eddahibi S, Adnot S, Carville C, Blouquit Y, Raffestin B. $\mathrm{L}$-arginine restores endothelium-dependent relaxation in pulmonary circulation of chronically hypoxic rats. $A m \mathcal{F}$ Physiol 1992;263:L1 194-200.

19 Wanstall JC, Hughes IE, O'Donnell SR. Evidence that nitric oxide from the endothelium attenuates inherent tone in isolated pulmonary arteries from rats with hypoxic pulmonary hypertension. Br f Pharmacol 1995;144:109-14.

20 Ohno M, Gibbons GH, Dzau VJ, Cooke JP. Shear stress elevates endothelial cGMP. Role of a potassium channel and G protein coupling. Circulation 1993;88:193-7.

21 Fukaya Y, Ohhashi T. Acetylcholine and flow-induced production and release of nitric oxide in arterial and venous endothelial cells. Am $\mathcal{f}$ Physiol 1996;270:H99-H106.

22 Zharikov S, Herrera H, Block ER. Hypoxic inhibition of L-arginine uptake by pulmonary endothelial cells (PEC): Correlation with changes in membrane potential. $\mathrm{Am}$ ) Respir Crit Care Med 1996;153:A573. 
23 Allen KM, Haworth SG. Impaired adaptation of pulmonary circulation to extrauterine life in newborn pigs exposed to 12 .

24 Meyrick B, Reid L. Endothelial and subintimal changes in rat hilar pulmonary artery during recovery from hypoxia. A quantitative ultrastructural study. Lab Invest 1980;42:603-15.

25 Hislop AA, Haworth SG. Pulmonary vascular damage in the development of cor pulmonale following hyaline membrane disease. Paediatric Pulmonol 1990;9:152-61

26 Heath D, Smith P, Rios DJ, Williams D, Harris P. Small pulmonary arteries in some natives of La Paz, Bolivia. Thorax 1981;36:599-604.

27 Steinhorn RH, Russell JA, Morin CFI. Disruption of cGMP production in pulmonary arteries isolated from fetal lambs with pulmonary hypertension. Am $\mathcal{f}$ Physiol with pulmonary
28 McQueston JA, Kinsella JP, Ivy DD, McMurtry IF, Abman $\mathrm{SH}$. Chronic pulmonary hypertension in utero impairs endothelium-dependent vasodilation. Am $\mathcal{f}$ Physiol 1995;268:H288-94.

29 Gao Y, Zhou H, Raj JU. Heterogeneity in role of endothelium-derived NO in pulmonary arteries and veins of full-term fetal lambs. Am f Physiol 1995;268:H1586-92.

30 Bansal V, Toga H, Raj RU. Tone dependent nitric oxide production in ovine vessels in vitro. Respir Physiol 1993;93:249-60.

31 Zhao Y, Packer CS, Rhoades RA. Pulmonary vein contracts in response to hypoxia. Am F Physiol 1993;9:L87-92.

32 Raj JU, Hillyard R, Kaapa P, Gropper M, Anderson J. Pulmonary arterial and venous constriction during hypoxia in 3 to 5 week old and adult ferrets. $\mathcal{F}$ Appl Physiol 1992;88:129-40. 